\title{
Comparison of Gas and Radiation Pressure in the Problems of the Dynamics of Planetary Nebulae
}

\author{
H. ZANSTRA \\ Sterrekundig Instituut, Roetersstraat, Amsterdam, the Netherlands
}

$\mathrm{W}^{\mathrm{s}}$ E may summarize Wilson's ${ }^{1}$ extended study of internal motions in planetary nebulae by stating that he finds the average expansion velocity in the regions whence originate the lines of $\mathrm{H}, \mathrm{NeIII}$, OIII to be roughly $20 \mathrm{~km} / \mathrm{sec}$, with rare examples ranging as low as $\mathrm{O}$ and as high as $50 \mathrm{~km} / \mathrm{sec}$. We ask whether radiation pressure and/or gas pressure may give, in an elementary calculation, velocities anywhere near these values.

\section{PLANETARIES FOR WHICH OPTICAL DEPTH IN LYMAN CONTINUUM, $\tau_{m} \sim 1$}

We define the standard planetary nebula as having electron density, $N_{e}=5 \times 10^{3}$; electron temperature, $T_{e}=10^{4} \mathrm{~K}$; and radius $r_{n}=10^{4}$ astronomical units. For this configuration, I find ${ }^{2}$ radiation pressure in the Lyman continuum gives an acceleration $\alpha_{c r}$ leading to a velocity of $3.2 \mathrm{~km} / \mathrm{sec}$ in $10^{4}$ years. Koelbloed's accurate solution for a nebula at rest and $\tau_{m}=1$ led to the ratio $\alpha_{r} / \alpha_{c r}=5.9$ at $\tau=0.5 \tau_{m}$ and 12.5 at $0.8 \tau_{m}$, where $\alpha_{r}$ is the acceleration due to Lyman- $\alpha$. (When expansion starts, the absorption moves off the line, and the acceleration drops. So the computed acceleration is decidedly an upper limit.)

Assuming a constant acceleration from rest and using the above values, we obtain the velocities $v_{c r}$ and $v_{r}$ in the first row of Table I. If we choose the value at $0.8 \tau_{m}$ rather than at $0.5 \tau_{m}, v_{r}$ would increase to 19.5 $\mathrm{km} / \mathrm{sec}$.

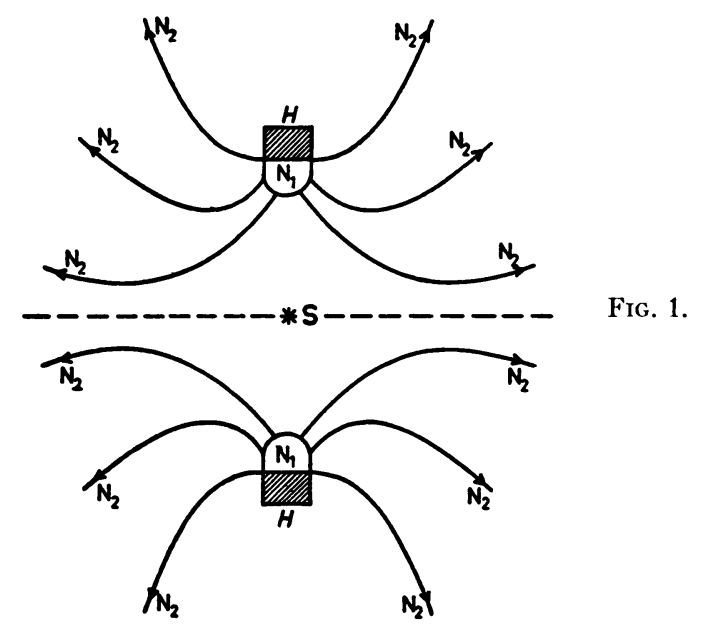

${ }^{1}$ O. C. Wilson, Astrophys. J. 111, 279 (1950).

2 H. Zanstra, Bull. Astron. Soc. Neth. 12, No. 465, 349 (1956).
If we consider a planetary for which $\tau_{m}=1$ and $T_{e}=10^{4}$, but allow arbitrary $N_{e}$ and $r_{n}$, then since we implicitly assume the same kind of central star, hence the same number of ionizing Lyman quanta, the total number of recombinations in the nebula remains constant. Thus, $N_{e}{ }^{2} r_{n}{ }^{3}$ remains constant. Also, $\alpha_{c r}$ is proportional to $N_{e}$. Thus substituting these relations in the expression for the velocity resulting from constant acceleration from rest, we scale the standard nebula up and down from the standard $r_{n}$ to obtain the results in the last two rows of Table I.

We estimate the expansion velocity resulting from gas pressure by assuming the nebula completely ionized, whence

$$
\alpha_{o}=-\frac{2 k T_{e}}{m_{\mathrm{H}}} \frac{d \ln N_{e}}{d r}
$$

and assume that over a distance equal to the nebular radius $N_{e}$ drops by a factor 2 . The result is in the sixth column of Table I; the corresponding time, in column seven. We conclude then that in our examples gas pressure is most important, and if the values of the various quantities used above are reasonable, we do indeed predict velocities near those observed.

\section{PLANETARIES FOR WHICH IN SOME DIRECTIONS $\tau_{m} \gg 1$}

A complete treatment would require a detailed consideration of what happens in the outer dark neutral hydrogen region along the lines of Oort's ${ }^{3}$ treatment for diffuse nebulae. Here, we give only some very simplified considerations. Contrary to what we thought at one time,${ }^{4}$ we do not suppose that the expansion velocities are due to an initial cataclysm, but consider a later stage in which there is a rather quiet development of the planetary.

TABLE I. Planetary nebula, with $L_{c}$ optical depth $\tau_{m} \simeq 1$ and at $\tau=0.5 \tau_{m}$, surrounding a central star.

\begin{tabular}{|c|c|c|c|c|c|c|}
\hline$N_{0} \mathrm{~cm}^{-\mathrm{s}}$ & $T_{\bullet}{ }^{\circ} \mathrm{K}$ & $r_{n}$ a.u. & $\begin{array}{c}\text { ver }_{\mathrm{c}} \\
\mathrm{km} / \mathrm{sec}\end{array}$ & $\begin{array}{c}y_{r} \\
\mathrm{~km} / \mathrm{sec}\end{array}$ & $\begin{array}{c}v_{o} \\
\mathrm{~km} / \mathrm{sec}\end{array}$ & $\begin{array}{c}t_{0} \\
\text { years }\end{array}$ \\
\hline $\begin{array}{r}5000 \\
40000 \\
625\end{array}$ & $\begin{array}{l}10000^{\circ} \\
10000^{\circ} \\
10000^{\circ}\end{array}$ & $\begin{array}{r}10000 \\
2500 \\
40000\end{array}$ & $\begin{array}{l}5.4 \\
7.6 \\
3.8\end{array}$ & $\begin{array}{r}13.4 \\
19.0 \\
9.5\end{array}$ & $\begin{array}{l}21 \\
21 \\
21\end{array}$ & $\begin{array}{r}4400 \\
1100 \\
18000\end{array}$ \\
\hline
\end{tabular}

${ }^{3}$ J. H. Oort, Bull. Astron. Soc. Neth. 12, No. 455, 177 (1955).

${ }_{4}$ H. Zanstra, Monthly Notices Roy. Astron. Soc. 93, 131 (1932). 
Photographs of planetary nebulae indicate that in most cases the luminous matter does not uniformly surround the star, so that one cannot have $\tau_{m} \gg 1$ in all directions. This is particularly clear for ring nebulae like that in Lyra, for which Wurm ${ }^{5}$ has given arguments that the $L_{c}$ absorption would be complete. These arguments only apply to the luminous ring, outside of which one would have a dark neutral hydrogen region, shown shaded in the schematical section of Fig. 1. Thus $\tau_{m} \gg 1$ for the direction $S \mathrm{H}$. This region of dark $\mathrm{H}$ prevents the Lyman- $\alpha$ escape outwards where $\tau \gg 1$; it is generated mostly in the part of the luminous ring for which $\tau=1$ and escapes in all directions through the regions of small $\tau$. For an order-ofmagnitude estimate of $v_{r}$ and $v_{g}$, we use the results of Sec. 1 and thus we expect the velocity of expansion to be a little over $20 \mathrm{~km} / \mathrm{sec}$. The observed value is $30 \mathrm{~km} / \mathrm{sec}$.

A more satisfactory derivation for the velocity gained by gas pressure is obtained by equating the mechanical energy gained in isothermal expansion to the kinetic energy of the matter, taking as average mass per particle $\frac{1}{2} m_{\mathrm{H}}$,

$$
\frac{1}{4} m_{\mathrm{H}} v_{0}^{2}=k T_{e} \ln \left(N_{1} / N_{2}\right) .
$$

${ }^{5} \mathrm{~K}$. Wurm, Contr. Obs. Astrof. U. di Padova in Asiage No. 73, p. 91 (1956).
We may consider a stationary outward flow of gas, as indicated by the arrows in Fig. 1, with initial concentration $N_{1}$ inside the luminous ring, and final concentration $N_{2}$. This is different from the situation in Sec. 1, where we assumed the whole nebula to expand. For an assumed $T_{e}=10^{4}{ }^{\circ} \mathrm{K}$, the observed $v=30 \mathrm{~km} / \mathrm{sec}$ would require $N_{1} / N_{2}=15$ according to Eq. (2), while $T_{e}=2.10^{4}$ ${ }^{\circ} \mathrm{K}$ would require $N_{1} / N_{2}=4$. These values do not seem unreasonable. If from ring to hole $N$ goes down by a factor 10 , and the thickness $L$ of the region goes up by a factor 10, the surface brightness inside the ring, proportional to $N^{2} L$, would be $1 / 10$ of that of the ring, which is about the ratio indicated by Aller's ${ }^{6}$ measurements from Minkowski's red photographs.

The faint outer envelope of the ring nebula in Lyra seems also to find a natural explanation: the emerging gases in Fig. 1 fan outward considerably beyond the bright ring and they remain excited there by the central star, since only the dark $\mathrm{H}$ ring intercepts the exciting radiation. Collision with the interstellar gas might then give bright edges to this envelope by compression, as was suggested by Minkowski, ${ }^{7}$ and the flow would stop there.

${ }^{6}$ L. H. Aller, Gaseous Nebulae (Chapman and Hall, London, 1956), pp. 56-57, Fig. III 5.

${ }_{7}$ R. Minkowski, Gas Dynamics of Cosmic Clouds, edited by H. C. van de Hulst and J. M. Burgers (North Holland Publishing Company, Amsterdam, the Netherlands, 1955), pp. 11-12.

\section{DISCUSSION}

R. MINKowSKI, Mount Wilson and Palomar Observatories, Pasadena, California: I would like to remark on Zanstra's idea of the ring nebula. The objects about which Wilson spoke are a highly selected group of very regular looking nebulae. Actually, there are many which do not fit such a simple picture at all and to which we refer in private talk as the Chamber of Horrors. We should expect to find in this Chamber of Horrors some sample of something like the ring nebula seen in a

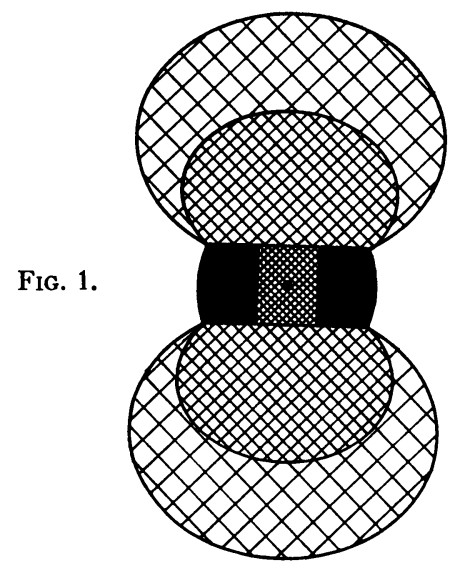

direction in the plane of the ring, the direction of Zanstra's diagram. We do not see anything like the very narrow ring of Zanstra's diagram, with matter coming out as shown. There is, however, a type of object which may very well represent the case of which Zanstra is thinking, and that would be a nebula which, in a very highly schematical way, would look about like Fig. 1. There are several examples which fit this scheme: NGC 40, NGC 650/651, GC 2371/ 2372 and others. It may be that Zanstra's ideas give the correct interpretation for at least this one class of objects in the Chamber of Horrors.

H. W. LIEPMANN, California Institute of Technology, Pasadena, California: I would suggest that we keep out of the Chamber of Horrors and stick with what looked to me the comparatively simple type of nebula discussed by Wilson. In fact, I think that in Wilson's model the aerodynamicists and the fluid dynamicists will eventually have a heyday because that looks like a very beautiful thing to argue about.

H. C. VAN DE HULST, Leiden Observatory, Leiden, Netherlands: If I understand well, Wilson has based the distribution of the various types of ions in the 
nebula entirely on the theory of the excitation; but if I remember well his earlier work, there were some discrepancies that did not quite fit that model, and that might remove the basis of your discussion. Do I gather correctly that the discrepancy has been resolved now or not?

O. C. WILson, Mount Wilson and Palomar Observatories, Pasadena, California: There is no real discrepancy. I showed you slitless pictures of 7662 , and there you could see the difference in image size. Now the only thing that is in the nature of a discrepancyand it is not really one-is that in some of the nebulae in the slitless pictures you cannot see differences of image size. The differences are too small to see. Wherever you can see them, they are always in this order, and I merely assume that for the ones that you cannot see, the zones are too close together to be resolved on the pictures.

H. C. VAN DE HulST: A second question. The velocities of expansion would be very simple to visualize if say the velocity of the $[\mathrm{NeV}]$ shell would be just proportional to the radius, say compared to the radius of the [OII] shell. Then everything would be expanding from the center, and you would need no forces at the present moment at all. I seem to gather from your further explanation that this is not true, but you cannot say observationally if there is any proportionality.

O. C. WILSON : Yes, you can say that there is not, because in other nebulae certain velocities become effectively zero. That is, if you see, say two nebulae, which are quite similar in their general proportions, one of them with velocity near zero for a particular ion and the other with $40 \mathrm{~km} / \mathrm{sec}$, it is a little bit hard to see how an initial velocity as small as zero could get you to an appreciable distance in the same time that a velocity of 40 or $50 \mathrm{~km} / \mathrm{sec}$ has gotten you there. I have thought of this point and I do not think it can be maintained.

H. W. LIEPMANN : From the aerodynamicist's viewpoint, the problem seems to be that we have a gas of many components, that we have to consider to a certain extent radiation pressure, and that we do not know exactly whether the gas expands into very high vacuum or whether there is some matter present. Is that correct?

O. C. WILson: Well, the only evidence is that the high excitation material is always on the inside, and the low excitation on the outside, so that if you are dealing with collisions they are not playing any appreciable roll in the excitation. There are no bright edges on these bright nebulae, such as one would expect from a collisional zone.

L. BIERMANN, Max Planck Institut für Physik, Göttingen, Germany: If I understand correctly, the matter in the shell is leaving the nebula, and from the dimensions, densities, and velocities, it should be possible just to see how much matter leaves, say in a million years.

\section{O. C. WILSON : I have not worked this out.}

G. C. MCVITTIE, University of Illinois, Urbana, Illinois: A problem which is fairly simply soluble, is one in which you have a pressure gradient plus gravitational attraction of the central star and a state of steady motion, but the snag is that it is necessary to have a supply of gas. Is it at all permissible to imagine that the central star is still ejecting? If so, then in the problem I have outlined curious things happen to the velocity of expansion, which rises to a maximum at some radius and then falls off again. What has been left out, of course, are the radiation problems which arise; that's an added difficulty. But would you contemplate the possibility of this kind of problem having any relevance?

O. C. WILSON: This actually is a point that has bothered me, because the nuclear stars very frequently in their spectra have emission bands indicating a breadth of the order of $75-100 \mathrm{~km} / \mathrm{sec}$. As in the case of novae, such breadths are generally attributed to ejection of material. However, I know of no observational evidence that these shells are not hollow. At the inner boundary, there is the highest excitation material; within that, so far as you can tell from the observations, there is nothing. My guess is that if there is any continuous ejection from the nuclei it is probably small.

\section{SPITZER, JR., Princeton University Observatory,} Princeton, New Jersey: As I remember, some time ago you analyzed the possibility of a steady state from the standpoint of seeing whether the different shells satisfied the equation of continuity, $\rho v r^{2}$ being constant. I remember that you came to a negative conclusion. Do you still feel that to be true?

O. C. WILSON: I thought at one time the central part might be filled with matter at a really high temperature, so that recombinations would be rare and you would not see any emission. But I have talked to Bowen about this, and he has pointed out that there are a whole series of ions which could serve as cooling agents, and there is no reason whatsoever to suppose that the temperature in the central regions is indeed 
high. Hence, one ought to see hydrogen emission, or helium and things like that, but we do not. Thus a continuity of mass flow seems doubtful.

H. ZANSTRA, Sterrekundig Instituut, Amsterdam C, Netherlands: I would reply to McVittie that certainly gravity cannot play any part in this problem of the planetary nebula because the nebula at present is so extremely far away from the star.

H. W. LIEPMANN : One of the problems which is rather interesting is the fact that the nebula is not centrally symmetric. Why is there ellipticity? I leave that for later discussion. 